\title{
Prenatal Development of the Human Central Nervous System, Normal and Abnormal
}

Kohei Shiota

\section{ABSTRACT}

The organogenesis of the central nervous system (CNS) begins during the third week of development, but its maturation requires a considerably long period of time until after birth. Therefore the developing nervous system is vulnerable to the deleterious effects of environmental factors during the pre- and perinatal periods. In addition, molecular studies have revealed various gene mutations that are responsible for congenital CNS disorders. This chapter provides an overview of the prenatal development of the human brain and spinal cord.

Keywords: Neurulation, Neuroectoderm, Neural tube defect, Histogenesis, Neuronal migration, Myelination.

How to cite this article: Shiota K. Prenatal Development of the Human Central Nervous System, Normal and Abnormal. Donald School J Ultrasound Obstet Gynecol 2015;9(1):61-66.

Source of support: Nil

Conflict of interest: None declared.

\section{EARLY DEVELOPMENT OF THE NERVOUS SYSTEM}

Major organogenesis in humans takes place mainly during the embryonic period by the end of the 8th week after fertilization (Fig. 1). The central nervous system (CNS) is derived from the ectodermal layer of the embryonic disk. By day 18 after fertilization, the midline of the embryonic disk deepens to form the neural groove. At the same time, the rostral part of the ectoderm thickens to form the symmetrical neural plate, which eventually forms the brain. The bilateral neural folds become conspicuous along the neural groove (Figs 2A and B). Around day 20, the neural folds begin to fuse with each other near the junction between the future brain and the spinal cord and the fusion extends both rostrally and caudally to form the neural tube (Fig. 2C). The rostral neuropore closes at about 24 days, and the caudal neuropore about 4 days later, which completes the formation of the neural tube (Fig. 2D). The site of final closure of the rostral neuropore is at the site of the embryonic lamina terminalis ( $\mathrm{O}^{\prime}$ Rahilly and Müller, 1999). The closure of the neural tube in human embryos is

\section{President}

Shiga University of Medical Science, Otsu, Shiga, Japan

Corresponding Author: Kohei Shiota, President, Shiga University of Medical Science, Otsu, Shiga-520-2192, Japan Phone: +81-775482000, e-mail: kohei.shiota@gmail.com generally described as a continuous process that begins at the level of the future cervical region and proceeds bidirectionally, but some investigators claim that neural tube closure in humans may initiate at multiple sites (Van Allen et al 1993; Nakatsu et al 2000).

Failure of neuropores to close can result in various forms of neural tube defects (NTDs). Cranial NTDs include anencephaly and encephalocele, and caudal NTDs spina bifida, myelomeningocele and meningocele (Figs 3A and B). NTDs are among the most common human malformations encountered in newborns.

By around day 20 (even before the closure of the neural tube), the primordia of three brain vesicles (forebrain or prosencephalon, midbrain or mesencephalon and rhombencephalon) can be identified as thickenings of the cranial neural ectoderm (Fig. 4A). Soon after the closure of the neural tube, it bends at three parts by the mesencephalic, pontine and cervical flexures (Figs 4A and B). The mesencephalic and pontine flexures are at the midbrain and hindbrain levels, respectively. The cervical flexure is located at the junction between the rhombencephalon and the spinal cord.

By the 5th week, the original three-part brain becomes subdivided into five parts. The forebrain gives rise to the telencephalon and the diencephalon (Figs $4 \mathrm{~B}$ and $\mathrm{C}$ ). The mesencephalon remains undivided and forms a tubular structure. With the development of the cerebellum and the pons, the division of the hindbrain into the metencephalon and the myelencephalon or medulla oblongata, becomes evident. The junction between the midbrain and hindbrain is relatively narrow and is known as the isthmus rhombencephali.

The telecephalic vesicles expand bilaterally to form the cerebral hemispheres. They enlarge rapidly and completely cover the diencephalon by the end of the embryonic period (Fig. 4D).

When the neural folds of both sides fuse with each other, some cells of the neural folds detach from the neuroectoderm and give off neural crest cells. These cells migrate extensively to generate a large diversity of differentiated cell types, including (1) the spinal cranial and autonomic ganglion cells, (2) the medulla of the adrenal gland, (3) the melanocytes of the epidermis, and (4) many of the skeletal and connective tissues of the head region. 


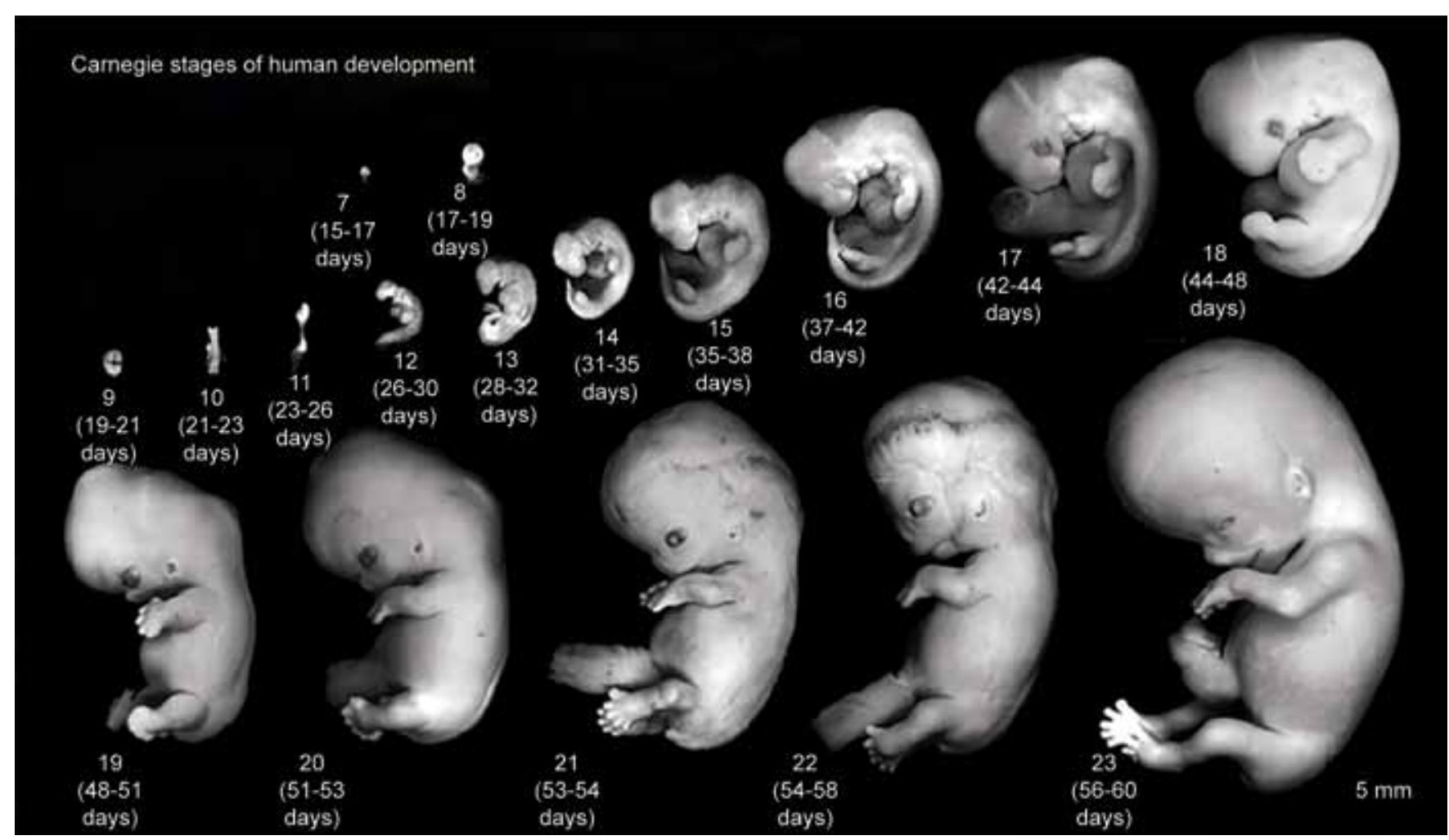

Fig. 1: Staged human embryos between the third and 8th weeks after fertilization (Carnegie stages 7-23) (Kyoto Collection of Human Embryos, Kyoto University)
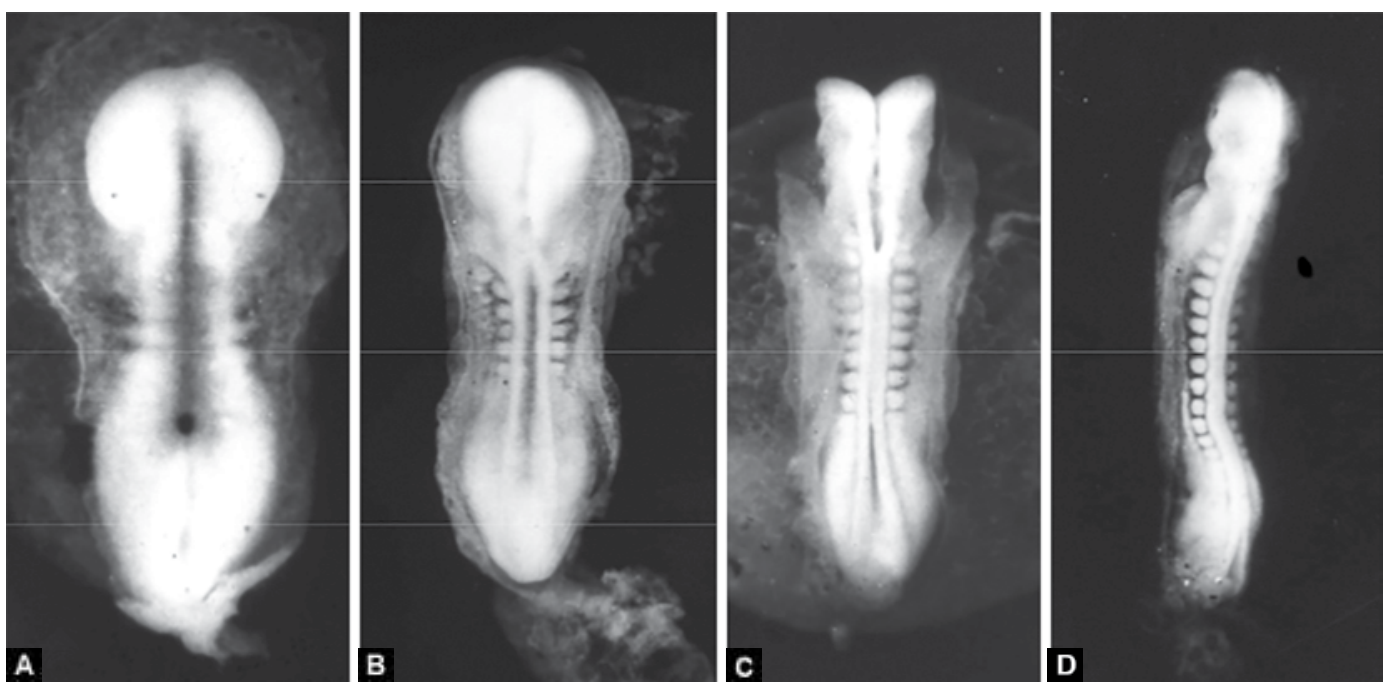

Figs 2A to D: Neurulation (neural tube formation) in human embryos: (A) 20 days after fertilization. Length $1.5 \mathrm{~mm}$, (B) 21 days. Length $2.1 \mathrm{~mm},(\mathrm{C}) 22$ days. Length $2.8 \mathrm{~mm}$ and (D) 24 days. Length $3.6 \mathrm{~mm}$ (Kyoto Collection of Human Embryos, Kyoto University)

\section{HISTOGENESIS IN THE DEVELOPING BRAIN}

Initially, the wall of the neural tube consists of a single layer of neuroepithelial cells, called the matrix layer (Fig. 5A). As this layer thickens, it gradually acquires the configuration of a pseudostratified epithelium. The nuclei of neuroectodermal cells become arranged in more and more layers, but all these cells remain in contact with the external and internal limiting membranes. Mitosis occurs near the ventricular surface of the cell layer, and differentiated early neuronal cells emigrate to the second layer closer to the outer surface of the neural tube (Fig. 5B). This mantle layer becomes progressively thicker as more cells migrate from the ventricular zone. The cells of the intermediate zone differentiate further into neurons and glial cells. Radial glial cells are present during early stages of neurogenesis. Neuronal cells emigrate from the mantle layer toward the outer surface of the neural tube along radial glia cells, where they form the cortical plate, a primordium of the cerebral cortex (Fig. 5C). The formation of the cortical plate takes place from 7 to 16 weeks and the outgrowth of the cerebral cortex and the proliferation and migration of cortical neurons largely occur during the fetal period. 


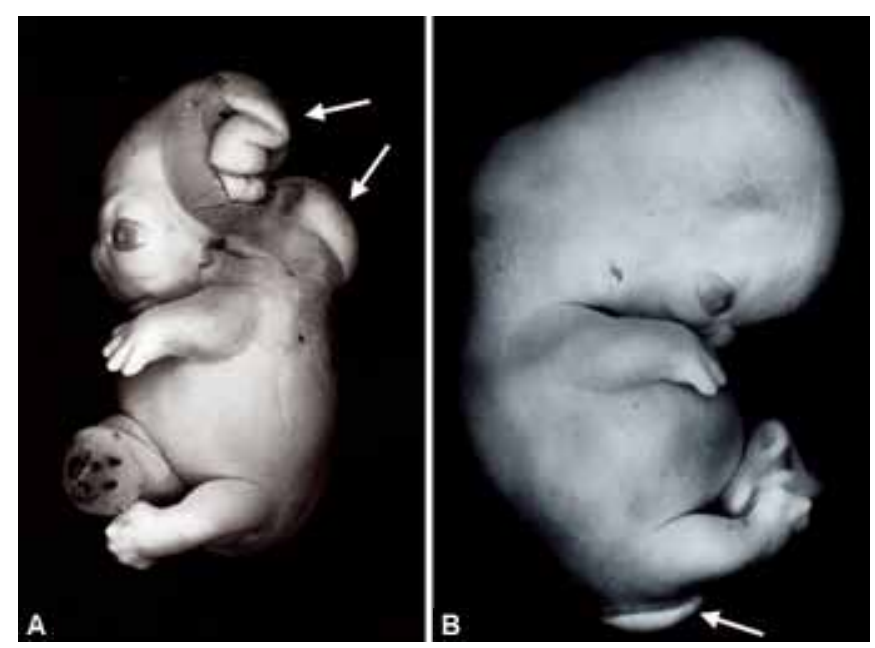

Figs 3A and B: Human embryos with neural tube defects (NTDs): (A) Cranial NTD. The brain tissue (arrows) is exposed, which will eventually become necrotic and result in anencephaly, (B) Caudal NTD. The exposed neural tissue (arrow) is overgrown and will result in myeloschisis or spina bifida aperta later in development (Kyoto Collection of Human Embryos, Kyoto University)

The detailed description of the brain histogenesis is beyond the scope of this paper, and readers may refer to the monographs by O'Rahilly and Mueller (1999) and ten Donkelaar et al (2014).

Birth defects due to abnormal cortical development may occur as a result of aberrant cell proliferation, neuronal migration and/or cortical organization. Defi- cient proliferation or excessive apoptosis of cortical neurons may lead to microcephaly or small brain. Various malformations of the brain may be produced due to abnormal migration of neuronal cells (neuronal migration disorders) and abnormal cortical organization (polymicrogyrias and schizencephalies).

\section{GROSS BRAIN DEVELOPMENT DURING THE FETAL PERIOD}

The most obvious changes in the fetal period are (1) outgrowth of the cerebellar hemispheres and the formation of its median part, the vermis, (2) continuous expansion of the cerebral hemispheres and the formation of the temporal lobe, sulci and gyri and (3) the formation of commissural connections, the corpus callosum in particular. Frontal, temporal and occipital poles and the insula become recognizable, whereas an olfactory bulb becomes visible on the ventral surface. Along with the rapid growth of the brain parts, the ventricular system also expands and becomes organized.

During the fetal period, the complex pattern of sulci and gyri arises (Figs 6A to E). On the lateral surface of the brain, the sulcus lateralis and the sulcus centralis can be recognized from the 4 th month. Owing to the development of the prefrontal cortex, the sulcus centralis gradually moves caudalwards. On its medial
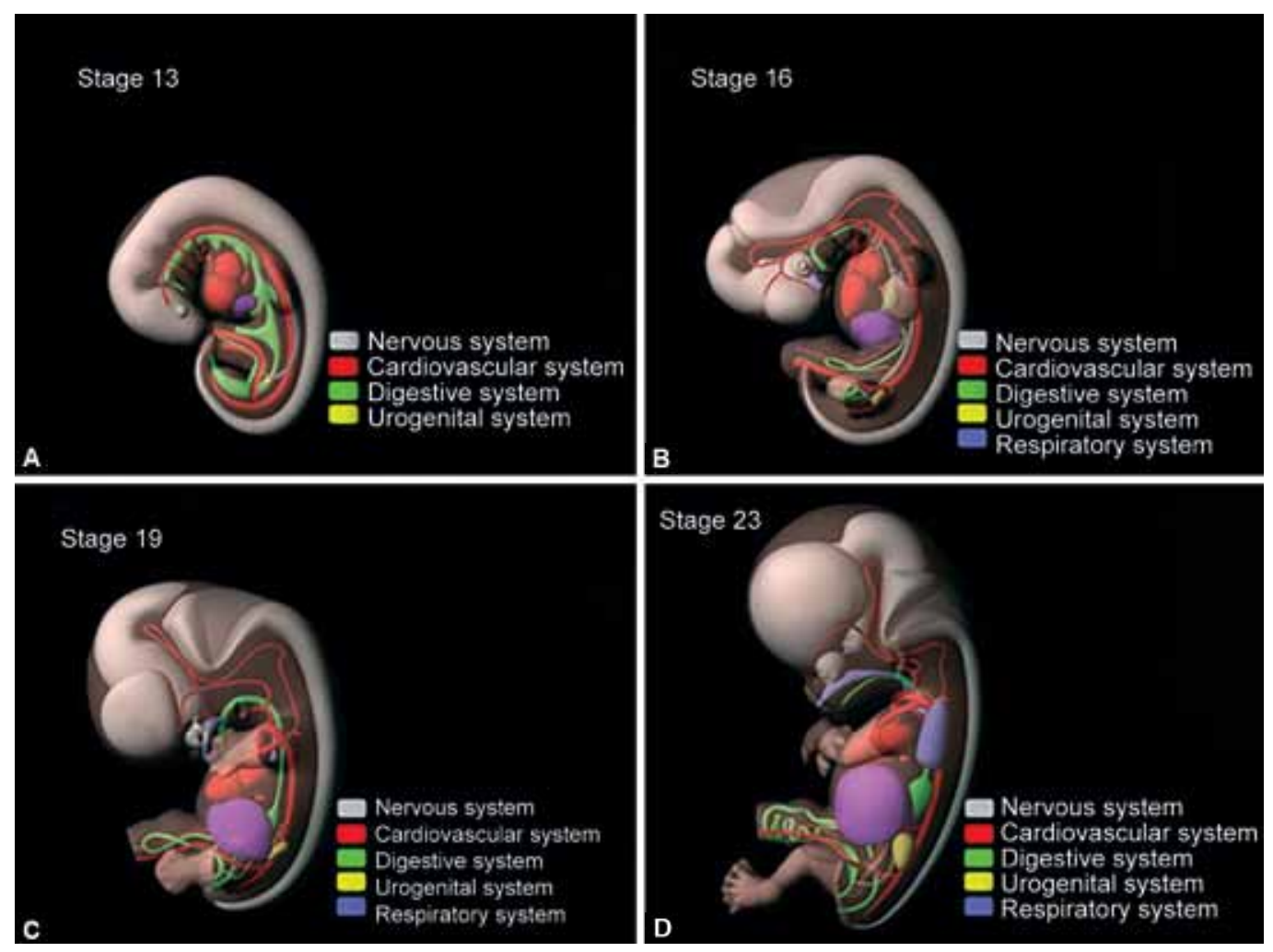

Figs 4A to D: Computer graphics models of human embryos illustrating the developing major internal organs (Yamada et al, 2006) 


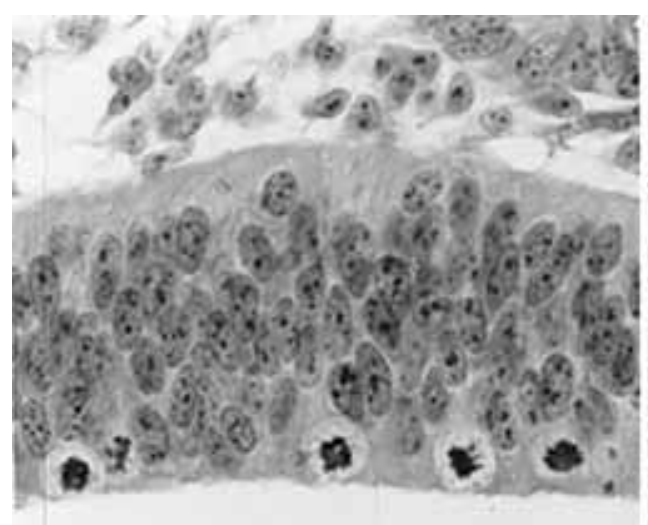

A

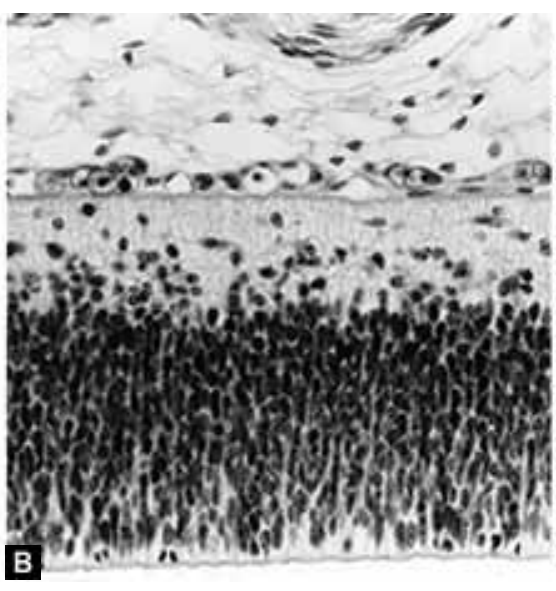

c

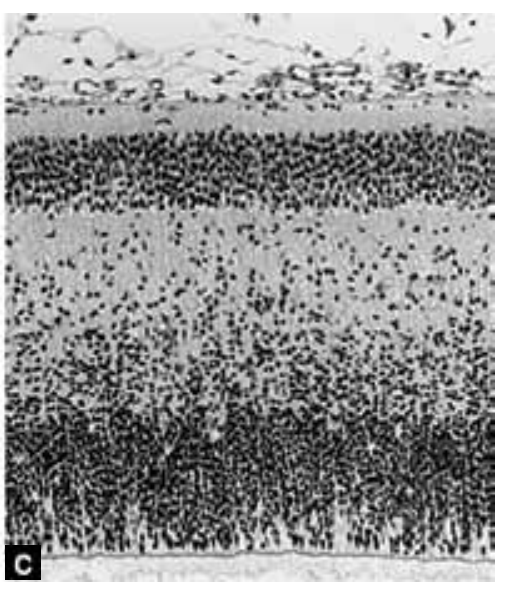

(B) 7 weeks, and (C) 11 weeks
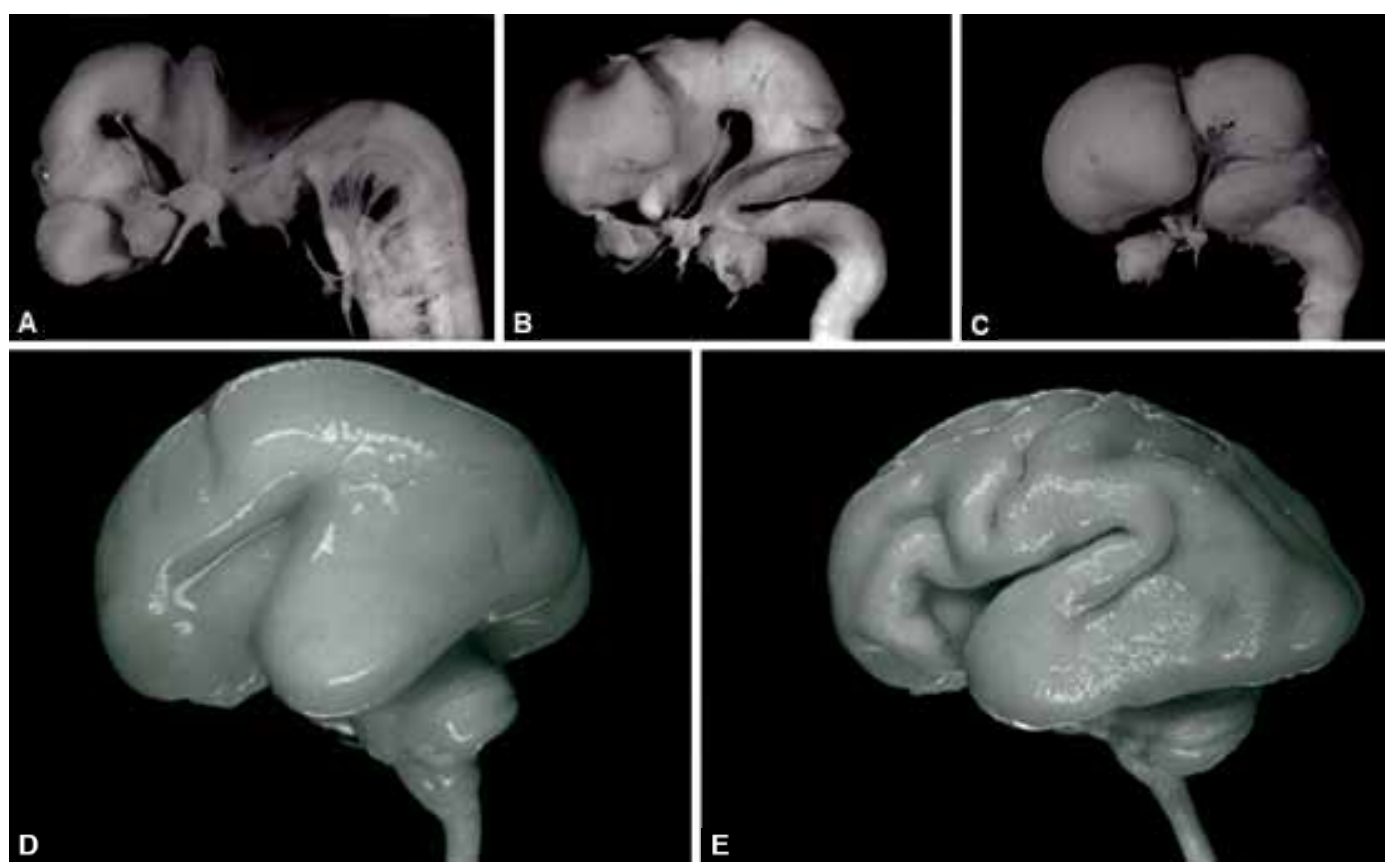

Figs 6A to E: Lateral views of the human embryonic and fetal brain: (A) 5 weeks after fertilization, (B) 7 weeks, (C) 8 weeks, and (D) 20 weeks and (E) 25 weeks (Kyoto Collection of Human Embryos, Kyoto University)

surface, the parieto-occipital and cingulate sulci first appear, followed by the calcarine and central sulci. The hippocampal formation comprises the dentate gyrus, the hippocampus, the subiculum and the parahippocampal gyrus.

The choroid plexus appears beneath the roof of the fourth ventricle and in the lateral ventricles during the 7 th week, and in the third ventricle early in the 8th week. The primordia of the choroid plexus appear as simple or club-shaped folds of ependymal cells protruding into the ventricles. The choroid plexuses become vascularized during the 8 th week of development. The embryonic choroid plexus is converted into the fetal type during the 9 th week as the embryonic capillary net is replaced by the loops of wavy capillaries lying under regular longitudinal epithelial folds.

\section{DEVELOPMENT OF THE SPINAL CORD}

The embryonic spinal cord can be divided into dorsal alar plates derived from lateral parts of the neural plate, and ventral basal plates derived from its medial parts (Fig.7A). The alar and basal plates are separated by the sulcus limitans (Fig. 7B). The bilateral alar plates are united by the roof plate, and the basal plates by the floor plate. The alar plates and incoming dorsal roots form the afferent or sensory part of the spinal cord, whereas the basal plate and its exiting ventral root form the efferent or motor part. The spinal ganglion cells arise from the neural crest.

Motor neurons and dorsal root ganglion cells appear in the embryonic spinal cord late in the 4th week. Dorsal root fibers enter the spinal gray matter early in development. The caudal end of the spinal cord reaches the end of the vertebral canal during the embryonic period, 


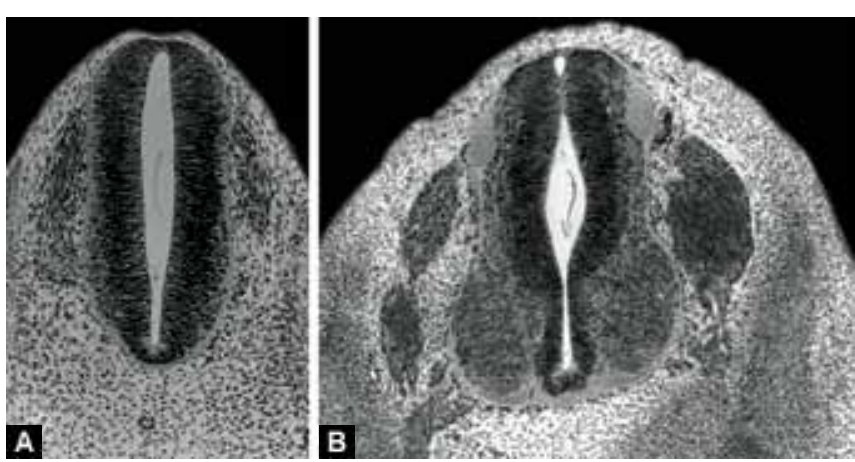

Figs 7A and B: Transverse sections of the human embryonic spinal cord: (A) 4 weeks after fertilization and (B) 6 weeks (Kyoto Collection of Human Embryos, Kyoto University)

whereas it 'ascends' to the sacral and later lumbar (L3-L4) levels as the spinal column elongates.

The most caudal part of the spinal cord is formed by a mechanism quite different from that of the cranial portion. The tissue that gives rise to the caudal spinal cord is a solid cord of cells in the caudal eminence and is called the medullary cord. This cord subsequently becomes cavitated and connected with the more rostrally located neural tube. It has been shown that the medullary cord gives rise to the spinal cord caudal to the level of the 32nd to 34th somites (Nievelstein et al, 1993). This process is called secondary neurulation and is distinguished from primary neurulation that forms the brain and the most part of the spinal cord by means of neural tube closure.

\section{DEVELOPMENT OF THE BLOOD SUPPLY OF THE BRAIN}

The brain is supplied by two pairs of internal carotid and vertebral arteries, connected by the circle of Willis. Along with the closure of the neural tube, primordial endothelial blood-containing channels are established, from which all other vessels in the brain are derived. In the fifth week, capital venous plexuses, the capital vein and three aortic arches are present. The internal carotid arteries develop early (4th week), followed by the posterior communicating artery, the caudal branch of the internal carotid, the basilar and vertebral arteries, the main cerebral arteries and finally the anterior communicating artery, thereby completing the circle of Willis. The major blood supply of the brain stem is first provided by the posterior communicating artery. Anastomotic channels unite the two longitudinal arteries, thereby initiating the formation of the basilar artery.

By 5 weeks, many of the definitive arteries are formed and transformed into the definitive pattern. By the end of the embryonic period, a network of leptomeningeal arteries arises from each middle cerebral artery and extends over the developing hemisphere. At 16 weeks of gestation, the anterior, middle and posterior cerebral arteries, contributing to the formation of the circle of Willis, are well established. The branching pattern of leptomeningeal arteries is completed by 28 weeks of gestation.

In younger premature newborns (born at 22-30 weeks of gestation), the blood vessels of the germinal, periventricular zone and the perforating ventriculopetal vessels are particularly vulnerable to perinatal asphyxia. Damage to these vessels often causes focal hemorrhagic lesions. In older premature infants (30-34 weeks), the fetal white matter is vulnerable to hypoxic-ischemic injury, leading to periventricular leukomalacia (PVL) and often resulting in infarction (necrosis) and cavitation.

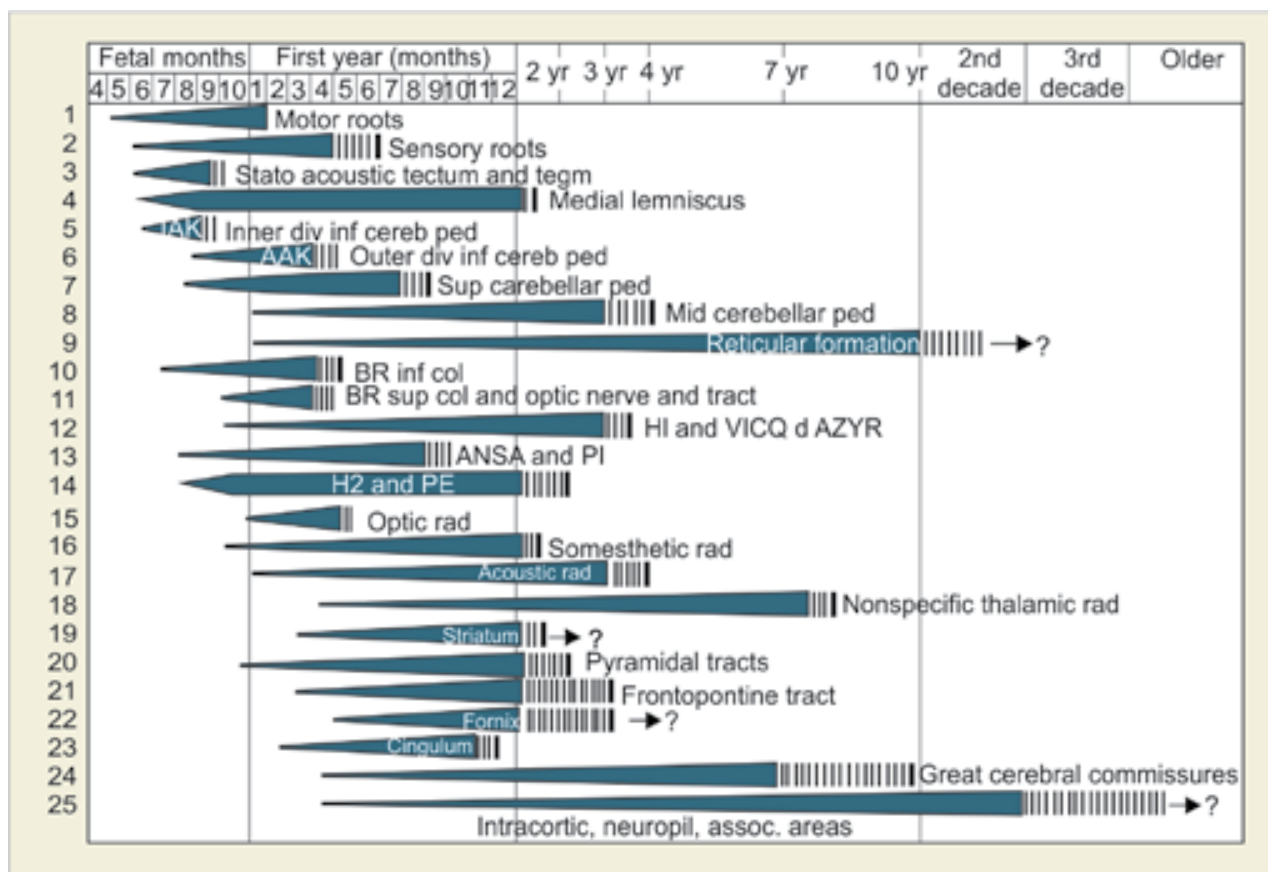

Fig. 8: Myelination of major fiber tracts in the human central nervous system (modified after Yakovlev and Lecours, 1967) 
Dural plexuses associated with the precardinal veins become modified to form the dural sinuses around the brain. Definitive venous channels emerge from the primitive vascular net during the later half of the embryonic period.

\section{MYELINATION}

The axons of fiber tracts need to be myelinated for proper functions. Myelination in the CNS is undertaken by oligodendrocytes, whereas that in the peripheral nervous system (PNS) depends on neurilemmal cells derived from the neural crest. Myelination is a rather slow process, and the earliest myelination is noted in the motor roots of the spinal cord around the 5th week of development and it proceeds caudorostrally (Fig. 8). In the brain stem, myelination starts at 8 th weeks of development. The vestibulospinal tracts become myelinated at the end of the second trimester, whereas the pyramidal tracts begin at the end of the third trimester. Myelination in these tracts is not completed until after birth, and the rate of myelin deposition is greatest during the first two postnatal years (van der Knaap and Valk 1990, 1995). Cortical association fibers are the last to become myelinated. It has been noted that the appearance of myelin in MRI lags about 1 month behind the histological events (van der Knaap and Valk 1995; Ruggieri 1997).

\section{REFERENCES}

1. Nakatsu T, Uwabe C, Shiota K. Neural tube closure in humans initiates at multiple sites: evidence from human embryos and implications for the pathogenesis of neural tube defects. Anat Embryol (Berl) 2000;201(6):455-466.

2. Nievelstein RA, Hartwig NG, Vermeij-Keers C, Valk J. Embryonic development of the mammalian caudal neural tube. Teratology 1993;48(1):21-31.

3. O'Rahilly R, Müller F. The embryonic human brain. An atlas of developmental stages. 2nd ed. Wiley-Liss, New York, 1999.

4. Ruggieri PM. Metabolic and neurodegenerative disorders and disorders with abnormal myelination. In: Ball WS (Editor). Pediatric Neuroradiology. Lippincott, Philadelphia, PA, pp 1997;175-237.

5. ten Donkelaar H, Lammens M, Hori A. Clinical neuroembryology 2nd ed. Springer, Berlin, Heidelberg and New York 2014.

6. van Allen MI, Kalousek DK, Chernoff GF, Juriloff D, Harris M, McGillivray BC, Yong SL, Langlois S, MacLeod PM, Chitayat D. Evidence for multi-site closure of the neural tube in humans. Am J Med Genet 1993;47(5):723-743.

7. van der Knaap MS, Valk J. MR imaging of the various stages of normal myelination during the first year of life. Neuroradiology 1990;31(6):459-470.

8. van der Knaap MS, Valk J. Magnetic Resonance of Myelin, Myelination and Myelin Disorders. 2nd ed. Springer, Berlin Heidelberg New York 1995.

9. Yakovlev PI, Lecours AR. The myelogenetic cycles of regional maturation of the brain. In: Minkowski A (Editor). Regional Development of the Brain in Early Life. Blackwell, Oxford, 1967. p. 3-70.

10. Yamada S, Uwabe C, Nakatsu-Komatsu T, Minekura Y, Iwakura M, Motoki T, Nishimiya K, Iiyama M, Kakusho K, Minoh $\mathrm{M}$ et al. Graphic and movie illustrations of human prenatal development and their application to embryological education based on the human embryo specimens in the Kyoto collection. Dev Dyn 2006;235(2):468-477. 